\title{
DIFFERENCE FILTER AND ITS APPLICATION IN SEPARATING PERIODIC COMPONENTS OF POLAR MOTION
}

\author{
Zheng-Xin LI Chang-Xia QIAN \\ Shanghai Observatory \\ Shanghai 200030, China
}

ABSTRACT. A new filtering method,in which the difference of second order is used to filter a time series of equal interval,and an example of its application on the separation of Annual and Chandler wobbles of polar motion are given in the paper.

\section{Basic Principal of the Difference Filter}

\subsection{Difference Filter of second order}

A simple harmonic motion is expressed as the follows (Ding et al.,1990):

$$
S(t)=A \sin (\omega t+\phi) .
$$

where $\mathrm{A}, \omega, \phi$ denote the amplitude,frequency, initial plirase of the motion. We also have the relation $w=2 \pi f=\frac{2 \pi}{T}$, where $\mathrm{f}$ is the linear frequency, $\mathrm{T}$ is the period.

If $t$ is restricted to be a positive integer, as $t=1,2, \cdots, n$, the $S(t)$ means a time series of equal interval. In order to simplify the recount in the text, it will be called a sine term series in the following.

For a sine term series, the differences of first and second order concerned with $\mathrm{S}(\mathrm{i})$ $(\mathrm{i}=2,3, \cdots, 11-1)$ are:

$$
\begin{gathered}
\Delta S(i-1)=S(i)-S(i-1)=2 \sin \frac{\omega}{2} A \cos \left(i \omega+\phi-\frac{\omega}{2}\right), \\
\Delta S(i)=S(i+1)-S(i)=2 \sin \frac{\omega}{2} A \cos \left(i \omega+\phi+\frac{\omega}{2}\right) . \\
\Delta^{2} S(i-1)=\Delta S(i)-\Delta S(i-1)=S(i+1)-2 S(i)+S(i-1) . \\
\Delta^{2} S(i-1)=-(2 \sin (\omega / 2))^{2} A \sin (i \omega+\phi)=-(2 \sin (\omega / 2))^{2} S(i) .
\end{gathered}
$$

we have:

$$
\begin{gathered}
S(i)+\Pi \Delta^{2} S(i-1)=0, \quad(i=2,3, \cdots, n-1) . \\
\Delta^{2} S(i-1)=-S(i) / \Pi . \\
\Pi^{2}=(2 \sin (\omega / 2))^{-2}
\end{gathered}
$$


The process of filtering, which is now define by relation (5), is called a Difference Filter of second order (DF). By means of the DF, any one of the sine term series, $S(i)$, can be eliminated by adding the product of the corresponding difference of second order $\Delta^{2} \mathrm{~S}(\mathrm{i}-1)$ and the factor $\mathrm{K}$.

The relation between $K$ and $\omega$ is shown in Figure 1. If $P$ denotes the time interval of neighbour sample points in composing a DF, the values $K$ related to the different sine term series, of which the periods are the products of $P$ and an integer or the reciprocal of an integer, are shown. It can be seen, when a value $K$ is used to filter a sine term through the DF, the other sine terms, which have the same $K$, will be filtered at the same time.

It can also be seen in the figure, for the sine terms, of which the periods equal $P$ divided by an integer $\left(\frac{P}{m} ; m=1,2, \cdots\right)$, their corresponding values of $\mathrm{K}$ equal $\infty$, their differences of second order vanish. So, there will be no distortion for these sine terms in passing the DF for which a limited value of $K$ is used to filtering other sine term.

\subsection{Difference Filter with Fidelity for a sine term}

Combine $S_{F}(t)$, which is supposed to be filtered, and $S_{R}(t)$, which is intended to be kept with no distortion, into a time series $f(t)$ :

$$
f(t)=S_{F^{\prime}}(t)+S_{R}(t), \quad(t=1,2, \cdots, n) .
$$

It is obvious to have the relation for their differences of second order:

$$
\Delta^{2} f(i-1)=\Delta^{2} S_{F}(i-1)+\Delta^{2} S_{R}(i-1), \quad(i=2,3, \cdots, n-1) .
$$

Compose the following DF in order to filter the $S_{F}(i)$ :

$$
\begin{gathered}
f(i)+\Pi_{F} \Delta^{2} f(i-1)=S_{R}(i)+\Pi_{F} \Delta^{2} S_{R}(i-1)+S_{F}(i)+K_{F} \Delta^{2} S_{F}(i-1) \\
=S_{R}(i)+\kappa_{F} \Delta^{2} S_{R}(i-1) .
\end{gathered}
$$

after using relation (6) we can have:

$$
\begin{gathered}
f(i)+\Pi_{F} \Delta^{2} f(i-1)=S_{R}(i) / Q_{R, F} . \\
Q_{R, F}=\Pi_{R} /\left(\Pi_{R}^{-}-\Pi_{F}\right) .
\end{gathered}
$$

The relation (10) shows, after passing the $D F$, which has been used to filter the $S_{F}(i)$, the original $S_{R}(i)$ term is distorted. The factor $Q_{R, F}$, which is now an index of the distortion, relates not only to the period to be filtered(represent by $\Pi_{F}$ ), but also to the period itself ( represent by $\kappa_{R}$ ). From (10), we can have the following relation:

$$
Q_{R, F}\left[f(i)+K_{F} \Delta^{2} f(i-1)\right]=S_{R}(i), \quad(i=2,3, \cdots, n-1) .
$$

From relation (12), we can define the DF, but with a multiplier of coefficient $Q_{R, F}$ now, as Difference Filter with Fidelity for a sine term or simply as Difference Filter with Fidelity (DFF), with which $S_{F}(i)$ is filtered but $S_{R}(i)$ still remain its existing with no distortion.

1.3 Difference Filter with Fidelity for a non-sine term 
For an arbitrary periodic function $F(t)$, it is always possible to use a trigonometric series to approach it.(Ding et al.,1990):

$$
F(t)=\sum_{j=1}^{\infty} A_{j} \sin \left(\omega_{j} t+\phi_{j}\right)=\sum_{j=1}^{\infty} S_{j}(t) .
$$

where frequency of the jth magnitude harmonic is $\omega_{j}=j \omega_{0}$, in which $\omega_{0}$ is the frequency of first harmonic; its corresponding period $T=\frac{2 \pi}{\omega_{0}}$ is the period of the $F(t)$.

Combine $F(t)$ and a sine term $N(t)$ into a time series $M(t)$ :

$$
M(t)=F(t)+N(t), \quad(t=1,2, \ldots, n) .
$$

Now we intend to filter the $N(t)$ but to keep $F(t)$ with no distortion, what kind of DF we should use?

It has already been mentioned during the discussing of Figure 1, if we choose the interval between the neighlour sample points to equal the period of a non-sine term(which is also the period $\mathrm{T}$ of $S_{1}(t)$ in relation (13)), the differences of second order for the harmonics will all vanish. So we have:

$$
\Delta^{2} F(i-1)=\sum_{i=1}^{\infty} \Delta^{2} S_{j}(i-1)=0 .
$$

Now we can compose the following DF:

$$
M(i)+\Pi_{N} \Delta^{2} M(i-1)=F(i)+\Pi_{N}^{-} \Delta^{2} F(i-1)+\left[N(i)+\Pi_{N} \Delta^{2} N(i-1)\right]=F(i) .
$$

from which we obtain the $F(t)$ series with no distortion. It is call a Difference Filter with Fidelity for non-sine term (DFF for non-sine term).

\subsection{Multiplicate Difference Filter with Fidelity}

When a time series to be treated contains more sine terms, a Multiplicate Difference Filter with Fidelity (MDFF) is supposed to be used. Establish:

$$
f_{0}(t)=C_{0}+C_{1} t+\sum_{L=1}^{m} S_{L}(t), \quad(t=1,2, \cdots, n) .
$$

in which there are a constant $C_{0}$, a linear term $C_{1} t$, and $m$ sine terms. It is possible to use a (m-1) step MDFF to keep one of the sine terms remains always the same without any distortion, but to eliminate all the other sine terms one by one in the whole process.

After filtering the first sine term of which the frequency is $\omega_{1}$, the new time series obtained $f_{1}(t) \quad(t=2,3, \cdots, n-1)$ is:

$$
\begin{aligned}
f_{1}(t)= & Q_{R .1}\left[f_{0}(t)+\Pi_{1} \Delta^{2} f_{0}(t-1)\right], \\
f_{1}(t)= & Q_{R, 1}\left[S_{1}(t)+\Pi_{1}^{-} \Delta^{2} S_{1}(t-1)\right]+Q_{R, 1}\left[S_{R}(t)+\Pi_{1} \Delta^{2} S_{R}(t-1)\right]+Q_{R, 1}\left(C_{0}+C_{1} t\right) \\
& +Q_{R, 1} \sum_{L=2}^{m}\left[S_{L}(t)+\Pi_{1} \Delta^{2} S_{L}(t-1)\right], \quad(L \neq R) \\
f_{1}(t)= & S_{R}(t)+Q_{R, 1}\left(C_{0}+C_{1} t\right)+Q_{R, 1} \sum_{L=2}^{m} \frac{S_{L}(t)}{Q_{L, 1}} ; \quad(L \neq R) .
\end{aligned}
$$


It can be seen in relation (18), there are the sine term $S_{R}(t)$, which is supposed to be k'pt with fidclity during the whole process, the constant and the linear torm but now with the multiplier of $Q_{R, 1}$, and the remaining (m-2) sine terms which are now with their multiplier of $\frac{Q_{R, 1}}{Q_{L, 1}} ; \quad(L=2,3, \cdots, m ; L \neq R)$.

Put the $f_{1}(t)$ into the next DFF:

$$
f_{2}(t)=Q_{R, 2}\left[f_{1}(t)+\Pi_{2} \Delta^{2} f_{1}(t-1)\right], \quad(t=3,4, \cdots, n-2) .
$$

and now the $f_{2}(t)$ obtained contains only $S_{R}(t)$, constant and linear term, and the remaining (m-3) sine terms.

Repeat the above process for altogether (m-1) times, we have:

$$
\begin{array}{cl}
f_{m-1}(t)=S_{R}(t)+B\left(C_{0}+C_{1} t\right), & (t=m, m+1, \cdots, n-m+1) . \\
B=\prod_{L=1}^{m} Q_{R, L} ; & (L \neq R) .
\end{array}
$$

It is easy to separate $S_{R}(t)$ from the time series (20) now.

When the time series intended to be kept, is a non-sine term $F(t)$, all the process will be the same. The only difference in the treatment is to use the period of $F(t)$ as the interval of neighbour sample points in using a Difference Filter(DF).

\section{A separation of Chandler and Annual term of polar motion in using Differ- ence Filter}

\subsection{Data of the polar motion}

The data used is the result of EOP(IERS) 90 C04 which has been pulblished by the IERS recently. The X,Y series of the result is the time series with 1-day interval from MJD43144.0 to M.JD48427.0, altogether 5284 sets of X,Y coordinates(IERS, 1991). The results have been smoothed before the publication with a factor of $\epsilon=10^{-2}$, so the periodic terms under 15 days are already elininated. A pre-treatment has been made in which the lower components, longer than 500 days, are eliminated by the method of digital filtering. The $\mathrm{X}(\mathrm{t}), \mathrm{Y}(\mathrm{t})$ series obtained are the results from which the Difference Filter is used to separate the Annual and Chandler Terms.

\subsection{Difference Filter used}

According to the evidence reported recently, it is quite possible that the Chandler term of polar motion can no more simply considered as a standard sine term(Gao,1991). For the purpose of theoretical studying, it is the time to treat both of the components as non-sine terms.

So, a MDFF non-sine term is used here to filter the $\mathrm{X}(\mathrm{t}), \mathrm{Y}(\mathrm{t})$ time series in order to separate the Annual or Chandler term with fidelity.

The $\mathrm{j}$ step of the filtering is $(\mathrm{j}=1,2,3, \cdots)$ :

$$
\begin{aligned}
& Z(i)+\Pi_{A_{j}} \Delta^{2} Z(i-1)=Z_{C(j+1)}(i), P_{C}=435 \text { days }(\text { Capitaine }, 1988), \\
& Z(i)+\Pi_{C_{j}} \Delta^{2} Z(i-1)=Z_{A(j+1)}(i), P_{A}=365 \text { days. }
\end{aligned}
$$


in which $\mathrm{Z}(\mathrm{i})$ represents the $\mathrm{X}(\mathrm{i})$ or $\mathrm{Y}(\mathrm{i}), P_{A}, P_{C}$ are the interval of the neighbour sample points, $K_{4, j}$ is the value of $K$ for the purpose of eliminating the jth magnitude harmonic of Annual term $\left(K_{A 1}=0.78429, K_{A 2}=0.28782, K_{A 3}=0.26358, \ldots\right)$, while $K_{C j}$ is the value of $K$ for eliminating the Chandler term $\left(K_{C_{1} 1}=1.07295, K_{C_{2}}=0.34972, K_{C 3}=0.25089, \cdots\right)$, $Z_{C^{\prime}(j+1)}(i), Z_{A(j+1)}(i)$ are the results after the filtering of the jth magnitude harmonic of the Chandler and Annual term. If we put it to the left side of the relation (22), a further step of filtering is begun.

\subsection{Result}

The above MDFF has repeated only once $(j=1,2)$, the $X, Y$ series of Chandler and Annial terms have converged. The results obtained are shown in Figures 2 and 3. A comparison is made in the right side of the figures of which the results were got by the conventional digital band filter. From the results shown in the figures, it is convinced that the using of Difference Filter is suscessful.

\section{Discussion}

(1) In using an ordinary DF, it is the standard sine term, expressed by (1), which may be filtered or kept with fidelity thoroughly. For a quasi-sine term, which is not so standard but still can be considered as a sine term in practice within the time interval covering the three neighbouring sample points, the above function of Difference Filter does exist, even the sine term being treated is not always so standard, having certain variation in its period, amplitude, or initial phrase.

(2) In the case of using DFF for a non-sine term, the remaining component (left side of Figures 2,3) is not the same in concept as those (right side of Figures 2,3) obtained by the ordinary filtering method: the former is the physical term itself which contains also the harmonics of higher magnitude: the latter is only the first harmonic of the physical term and can do nothing on the higher harmonics.

(3) The advantage of difference filter also includes the simplicity of the process, the amount of the calculation is also very small. Its CPU time is smaller than the ordinary method in several magnitudes.

\section{Reference:}

Capitaine N. et al., Astron. Astrophys., 202(1988). 306

Ding, Yue-Rong et al., Method In Processing For Astronomical Observational Data, Press of Nan.Jing University, 1990

Gao, Yu-ping, A Study of Polar Motion From The Observational Data of Neu Techniques, Thesis of Master degree,Shanghai Observatory,1991

IERS, Annual Report. For 1990, Paris Observatory, 1991

Li, Zheng-Xin, Annals of Shanghai Observatory, No.2, 38-47, 1980

Wu, Shou-Xian et al., Publications of the Shaanxi Astronomical Observatory, 3, 33-38, 1980 
Fig. 1 The relation between $K$ and $\omega$

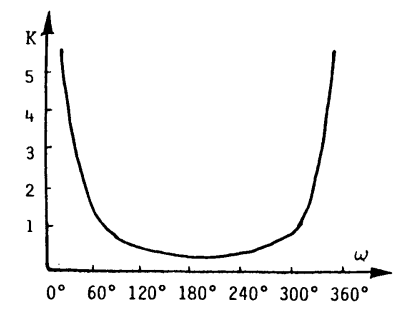

$\infty \quad 6 p \quad 3 p \quad 2 p \quad 1.5 p \quad 1.2 p \quad p$

$\begin{array}{lllllll}\frac{\mathrm{p}}{1} & \ldots & \cdots & \frac{2 \mathrm{p}}{3} & \cdots & \ldots & \frac{\mathrm{p}}{2}\end{array}$

$\begin{array}{lllllll}\frac{p}{2} & \cdots & \cdots & \frac{2 p}{5} & \cdots & \cdots & \frac{p}{3}\end{array}$

: $:$ : : : :

$\frac{p}{m-1} \cdots \cdots \frac{2 p}{2 m-1} \cdots \cdots \frac{p}{m}$
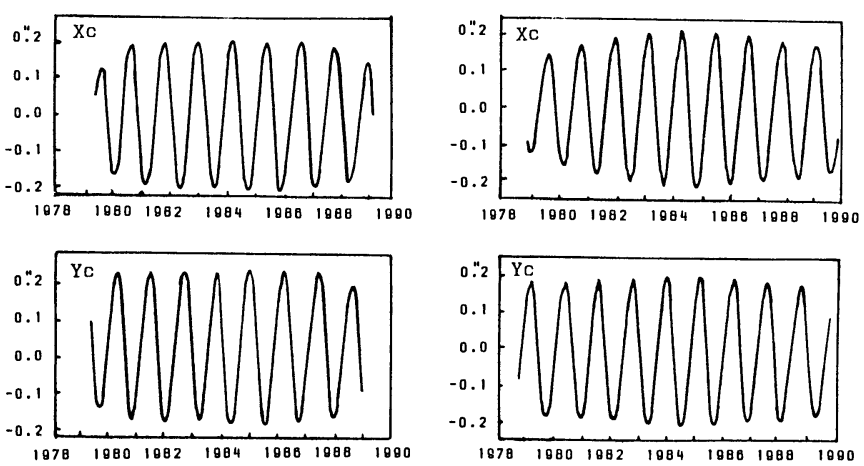

Fig. 2 Chandler components from 1979 through 1990
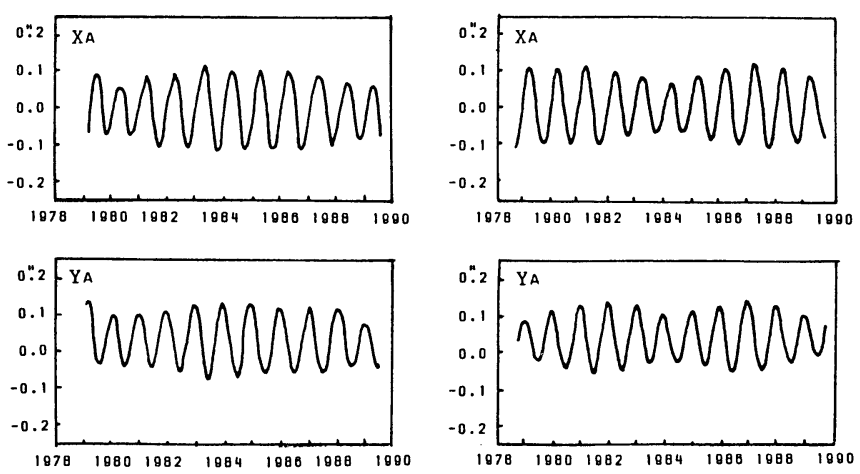

Fig.3 Annual components from 1979 through 1990 